Applied Mathematical Sciences, Vol. 7, 2013, no. 56, 2769 - 2775

HIKARI Ltd, www.m-hikari.com

\title{
Close-to-Convex Functions Defined by Fractional Operator
}

\author{
Melike Aydog̃an \\ Department of Mathematics \\ Işık University, Meşrutiyet Koyu, Şile İstanbul, Turkey \\ melike.aydogan@isikun.edu.tr \\ Yasemin Kahramaner \\ Department of Mathematics \\ İstanbul Ticaret University, İstanbul, Turkey \\ Yaşar Polatog̃lu \\ Department of Mathematics and Computer Sciences \\ İstanbul Kültür University, İstanbul, Turkey
}

Copyright (C) 2013 Melike Aydog̃an et al. This is an open access article distributed under the Creative Commons Attribution License, which permits unrestricted use, distribution, and reproduction in any medium, provided the original work is properly cited.

\begin{abstract}
Let $S$ denote the class of functions $f(z)=z+a_{2} z^{2}+\ldots$ analytic and univalent in the open unit disc $D=\{z \in \mathbb{C}|| z \mid<1\}$. Consider the subclass and $S^{*}$ of $S$, which are the classes of convex and starlike functions, respectively. In 1952, W. Kaplan introduced a class of analytic functions $f(z)$, called close-to-convex functions, for which there exists $\phi(z) \in \mathbb{C}$, depending on $f(z)$ with $\operatorname{Re}\left(\frac{f^{\prime}(z)}{\phi^{\prime}(z)}\right)>0$ in , and prove that every close-to-convex function is univalent. The normalized class of close-to-convex functions denoted by $K$. These classes are related by the proper inclusions $C \subset S^{*} \subset K \subset S$.

In this paper, we generalize the close-to-convex functions and denote $K(\lambda)$ the class of such functions. Various properties of this class of functions is alos studied.
\end{abstract}

Mathematics Subject Classification: 30C45, 30C55 
Keywords: Starlike, convex, close-to-convex, fractional calculus

\section{Introduction}

Let be the family of functions $p(z)=1+\sum_{n=1}^{\infty} p_{n} z^{n}$ which are analytic in $D$ and satisfy the conditions $p(0)=1, \operatorname{Rep}(z)>0$ for all $z \in$.

Let $S$ denote the class of functions $f(z)$ of the form $f(z)=z+\sum_{n=2}^{\infty} a_{n} z^{n}$ which are analytic and univalent in $D$.

We recall here the definition of the well-known classes of starlike, convex and close-to-convex functions [3], respectively,

$$
\begin{gathered}
S^{*}=\left\{f \in S \mid R e \frac{z f^{\prime}(z)}{f(z)}>0, z \in D\right\}, \\
C=\left\{f \in S \mid\left(1+\operatorname{Re} \frac{z f^{\prime \prime}(z)}{f^{\prime}(z)}\right)>0, z \in D\right\}, \\
K=\left\{f \in S \mid \exists \psi \in C, \operatorname{Re} \frac{f^{\prime}(z)}{\psi(z)}>0, z \in D\right\} .
\end{gathered}
$$

When considering these definitions above, in general, the functions belonging to them can be represented as the functions of .

Alexander's Theorem says us "if $\phi(z)$ is convex, then $\psi(z):=z \phi^{\prime}(z)$ is starlike". Hence, we can rewrite $K$ as follows:

$$
K=\left\{f \in S \mid \exists \psi \in S^{*} \ni \operatorname{Re}\left(z \frac{f^{\prime}(z)}{\psi(z)}\right)>0, \text { forall } z \in D\right\} .
$$

A fairly complete treatment, wtih applications of the fractional calculus, is given in the books [6] by Oldham and Spanier, and [5] by Miller and Ross. We refer to [10] for more insight into the concept of the fractional calculus. For further details on the materials in this paper see [4].

For convenience, we shall remind some definitions of the fractional calculus (i.e, fractionla integral and fractional derivative).

The fractional integral of order $\lambda$ for ana analytical function $f(z)$ in $D$ is defined by

$$
D_{z}^{-\lambda} f(z)=\frac{1}{\Gamma(\lambda)} \int_{0}^{z} \frac{f(\zeta)}{(z-\zeta)^{1-\lambda}} d \zeta,(\lambda>0)
$$


where the multiplicity of $(z-\zeta)^{\lambda-1}$ is removed by requiring $\log (z-\zeta)$ to be real when $(z-\zeta)>0$.

The fractional derivative of order $\lambda$ for an analytic function $f(z)$ in $D$ is defined by

$$
D_{z}^{\lambda} f(z)=\frac{d}{d z}\left(D_{z}^{-\lambda} f(z)\right)=\frac{1}{\Gamma(1-\lambda)} \frac{d}{d z} \int_{0}^{z} \frac{f(\zeta)}{(z-\zeta)^{\lambda}} d \zeta,(0 \leq \lambda<1),
$$

where the multiplicity of $(z-\zeta)^{-\lambda}$ is removed by requiring $\log (z-\zeta)$ to be real when $(z-\zeta)>0$.

Under the hypothesis of the fractional derivative , the fractional derivative of order $(n+\lambda)$ for an analytic function $f(z)$ in $D$ is defined by

$$
D_{z}^{n+\lambda} f(z)=\frac{d^{n}}{d z^{n}}\left(D_{z}^{\lambda} f(z)\right),\left(0 \leq \lambda<1, n \in N_{0}=\{0,1,2, \ldots\}\right) .
$$

From the definitions of the fractional calculus, we see that

$$
\begin{gathered}
D_{z}^{-\lambda} z^{k}=\frac{\Gamma(k+1)}{\Gamma(k+1+\lambda)} z^{k+\lambda},(\lambda>0, k>0) \\
D_{z}^{\lambda} z^{k}=\frac{\Gamma(k+1)}{\Gamma(k+1+\lambda)} z^{k-\lambda},(0 \leq \lambda<1, k>0)
\end{gathered}
$$

and

$$
D_{z}^{n+\lambda} z^{k}=\frac{\Gamma(k+1)}{\Gamma(k+1-\lambda)} z^{k-n-\lambda},\left(0 \leq \lambda<1, k>0, n \in N_{0}, k-n \neq-1,-2, \ldots\right)
$$

Therefore we see that for any real $\lambda$

$$
D_{z}^{\lambda} z^{k}=\frac{\Gamma(k+1)}{\Gamma(k+1-\lambda)} z^{k-\lambda},(k>0, k-\lambda \neq-1,-2, \ldots)
$$

\section{Main Results}

Using the rule of the fractional derivative which is mentioned in the preceding, we define the $\lambda$ - fractional operator as follows,

$$
\begin{gathered}
f(z)=z+a_{2} z^{2}+\ldots+a_{n} z^{n}+\ldots \Rightarrow D_{z}^{\lambda} f(z)=D_{z}^{\lambda}\left(z+a_{2} z^{2}+\ldots+a_{n} z^{n}+\ldots\right) \\
D^{\lambda} f(z)=\Gamma(2-\lambda) z^{\lambda} D_{z}^{\lambda} f(z)=z+\sum_{n=2}^{\infty} a_{n} \frac{\Gamma(2-\lambda) \Gamma(n+1)}{\Gamma(n+1-\lambda)} z^{n}
\end{gathered}
$$

From the definition of $D^{\lambda} f(z)$ we have the following properties. 
i.

$$
D^{\prime} f(z)=D f(z)=\lim _{\lambda \rightarrow 1} D^{\lambda} f(z)=z f^{\prime}(z)
$$

ii.

$$
\begin{gathered}
D^{\lambda}\left(D^{\delta} f(z)\right)=D^{\delta}\left(D^{\lambda} f(z)\right)= \\
z+\sum_{n=2}^{\infty} a_{n} \frac{\Gamma(2-\lambda) \Gamma(2-\delta)(\Gamma(n+1))^{2}}{\Gamma(n+1-\lambda) \Gamma(n+1-\delta)} z^{n}
\end{gathered}
$$

iii.

$$
\begin{gathered}
D\left(D^{\delta} f(z)\right)=z+\sum_{n=2}^{\infty} a_{n} n a_{n} \frac{\Gamma(2-\lambda) \Gamma(n+1)}{\Gamma(n+1-\lambda} z^{n}=z\left(D^{\delta} f(z)\right)^{\prime}= \\
\Gamma(2-\lambda) z^{\lambda}\left(\lambda D_{z}^{\lambda}+z D_{z}^{\lambda+1} f(z)\right) ;
\end{gathered}
$$

vi.

$$
\begin{gathered}
\frac{D\left(D^{\lambda} f(z)\right)}{D^{\lambda} f(z)}=z \frac{f^{\prime}(z)}{f(z)}, \text { for } \lambda=0, \\
=1+z \frac{f^{\prime \prime}(z)}{f^{\prime}(z)}, \text { for } \lambda=1 .
\end{gathered}
$$

Thus, we define the following class of functions.

Definition 2.1 Let $f(z)=z+\sum_{n=2}^{\infty} a_{n} z^{n}$ be an element of $S$. Then $f(z)$ is said to be $\lambda$-fractional close-to-convex function in $D$ if there exists a function $g(z)$ of $S^{*}$ such that

$$
\operatorname{Re}\left(\frac{D\left(D^{\lambda} f(z)\right)}{g}(z)\right)>0
$$

for all $z \in D$. The class of these functions is denoted by $K(\lambda)$.

It is obviously that $K(0)=K$.

By using the definition above and properties of $\lambda$ - fractional operator $D^{\lambda} f(z)$, we have the following properties.

i.

$$
\begin{gathered}
L(z)=\frac{z}{1-z}=z+z^{2}+\ldots+z^{n}+\ldots D^{\lambda} L(z)=z+\sum_{n=2}^{\infty} \frac{\Gamma(2-\lambda) \Gamma(n+1)}{\Gamma(n+1-\lambda)} z^{n}= \\
z F(2,1,2-\lambda ; z) .
\end{gathered}
$$


Then we have,

$$
\begin{gathered}
\operatorname{Re}\left(\frac{D\left(D^{\lambda} f(z)\right)}{g(z)}\right)>0 \Rightarrow \operatorname{Re}\left(\frac{z f^{\prime}(z) * D^{\lambda} L(z)}{g(z)}\right)>0 \Rightarrow \\
\operatorname{Re}\left(\frac{z f^{\prime}(z) * z F(2,1,2-\lambda ; z)}{g(z)}\right)>0 .
\end{gathered}
$$

(a) For $\lambda=0$,

$$
\operatorname{Re}\left(\frac{z f^{\prime}(z) * L(z)}{g(z)}\right)>0 \Rightarrow \operatorname{Re}\left(\frac{z f^{\prime}(z)}{g(z)}\right)>0 .
$$

(b) For $\lambda=1$,

$$
\operatorname{Re}\left(\frac{z f^{\prime}(z) * L^{\prime}(z)}{g(z)}\right)=\operatorname{Re}\left(\frac{z f^{\prime}(z)}{g(z)}\right)>0 .
$$

Where $k(z)$ is a Koebe function.

ii.

$$
\begin{aligned}
\operatorname{Re}\left(\frac{D\left(D^{\lambda} f(z)\right)}{g}(z)\right) & >0=\operatorname{Re}\left(z \frac{f^{\prime}(z)}{g(z)}\left(1+z \frac{f^{\prime \prime}(z)}{f^{\prime}(z)}\right)\right)>0, \lambda=1 \\
= & \operatorname{Re}\left(z \frac{f^{\prime}(z)}{g(z)}\right)>0, \lambda=0 .
\end{aligned}
$$

Theorem 2.2 Let $f(z)$ be an element of $K(\lambda)$. Then

$$
\frac{r(1-r)}{(1+r)^{3}} \leq\left|D\left(D^{\lambda} f(z)\right)\right| \leq \frac{r(1+r)}{(1-r)^{3}}
$$

Proof 2.3 Using the definition of the class $K(\lambda)$, we can write

$$
\frac{D\left(D^{\lambda} f(z)\right)}{g(z)}=p(z) \Rightarrow D\left(D^{\lambda} f(z)\right)=p(z) g(z) .
$$

where $p(z) \in P$. On the other hand, we have the inequalities

$$
\frac{1-r}{1+r} \leq|p(z)| \leq \frac{1+r}{1-r}
$$

and

$$
\frac{r}{(1+r)^{2}} \leq|g(z)| \leq \frac{r}{(1-r)^{2}}
$$

from [1]. By considering (14), (15) and (16), we obtain (13). 
If $f(z)$ be an element of $K(\lambda)$. Then

$$
\begin{gathered}
\frac{1-r}{r(1+r)^{3}} \leq\left|f^{\prime}(z)\right| \leq \frac{(1+r)}{r(1-r)^{3}}, \text { for } \lambda=0, \\
\frac{(1-r)}{(1+r)^{3}} \leq\left|f^{\prime}(z)+z f^{\prime \prime}(z)\right| \leq \frac{(1+r)}{(1-r)^{3}}, \text { for } \lambda=1 .
\end{gathered}
$$

Theorem 2.4 Let $f(z)$ be an element of $K(\lambda)$; then

$$
\left|a_{n}\right| \leq \frac{n \Gamma(n+1-\lambda)}{\Gamma(2-\lambda) \Gamma(n+1)} z^{n}
$$

We notice that this result is, indeed, sharp since the extremal function

$$
f(z)=z+\sum_{n=2}^{\infty} \frac{n \Gamma(n+1-\lambda)}{\Gamma(2-\lambda) \Gamma(n+1)} z^{n}
$$

is the solution of the fractional differential equation

$$
D_{z}^{\lambda} f(z)=\frac{1}{\Gamma(2-\lambda)} z^{-\lambda} \frac{z}{(1-z)^{2}}
$$

Proof 2.5 If we use the definition of the class $K(\lambda)$, then we can write

$$
\begin{gathered}
\frac{D\left(D^{\lambda} f(z)\right)}{g(z)}=p(z) \Rightarrow D\left(D^{\lambda} f(z)\right)=p(z) g(z) \Rightarrow \\
z+\sum_{n=2}^{\infty} \frac{\Gamma(2-\lambda) \Gamma(n+1)}{\Gamma(n+1-\lambda) n a_{n} z^{n}} \\
=\left(z+b_{2} z^{2}+\ldots+b_{n} z^{n}+\ldots\right)\left(1+p_{1} z+p_{2} z^{2}+\ldots+p_{n} z^{n}+\ldots\right) \Rightarrow \\
n a_{n} \frac{\Gamma(2-\lambda) \Gamma(n+1)}{\Gamma(n+1-\lambda)}=\left(b_{n}+b_{n-1} p_{1}+\ldots+b_{2} p_{n-2}+b_{1} p_{n-1}\right) \Rightarrow \\
n\left|a_{n}\right| \frac{\Gamma(2-\lambda) \Gamma(n+1)}{\Gamma(n+1-\lambda)} \leq\left|b_{n}\right|+\left|b_{n-1}\right|\left|p_{1}\right|+\ldots+\left|b_{1}\right|\left|p_{n-1}\right| \Rightarrow \\
\leq n+(n-1) 2+(n-2) 2+\ldots+2.2+1.2 \\
=n+2[1+2+\ldots+(n-1)]=n^{2} \Rightarrow \\
\left|a_{n}\right| \leq \frac{n \Gamma(n+1-\lambda)}{\Gamma(2-\lambda) \Gamma(n+1)}
\end{gathered}
$$

We notice that if we take $\lambda=0$ then we obtain $\left|a_{n}\right| \leq n$ which is the coefficient inequality for the close-to-convex functions, and we take $\lambda=1$, then $\left|a_{n}\right| \leq 1$. 


\section{References}

[1] Duren, P. , Univalent Functions, Springer Verlag, New York, Berlin, Heidelberg, Tokyo, (1983).

[2] Golusin , Geometrische Funktionentheorie, German Edition, DVW, Berlin, (1957).

[3] Kaplan, W. , Close-to-convex schlicht functions, Mich. Math. J. , pp. 169-185, (1952).

[4] Kilbas, A. A., Srivastava, H. M. , and Trujillo, J. J., Theory and Applications of Fractional Differential Equations, Elsevier Publ. , North-Holland Mathematics Studies, Vol. 204, (2006).

[5] Miller, K. S. , and Ross, B. , An Introduction to the Fractional Calculus and Fractional Differential Equations, John Wiley and Sond, Inc. , New York, 1993.

[6] Oldham, K. B. , and Spanier, J. , The Fractional Calculus, Academic Press, 1974.

[7] Owa, S. ,On the Distortion Theorems I, Kyungpook Math. J. , 18 (1978), pp. 53-59.

[8] Owa, S. and Srivastava, H. M. , Univalent and Starlike Generalized Hypergeometric Functions, Canad. J. Math. , 39 (1987), 1057-1077.

[9] Reade, M. O. , On close-to-convex functions, Mich. Math. J. , 3 (1955), 59-62.

[10] Srivastava, H. M. , and Owa, S. Univalent Functions, Fractional Calculus and Their Applications, Jhon Wiley and Sons, New York, 1989.

Received: March 5, 2013 